\title{
SOBRE LA COLECCIÓN DE ALGAS DEL HERBARIO DE LA SOCIEDAD MALAGUEÑA DE CIENCIAS (S. XIX)
}

\author{
Francisco CONDE POYALES
}

RESUMEN. A través del trabajo de Bellón, 1940, descubrimos en el Laboratorio Oceanográfico de Málaga, parte de lo que fuera el herbario de la Sociedad Malagueña de Ciencias (HSMC). En dicho herbario, de 242 pliegos de algas, encontramos material de casi un siglo y medio de antigüedad, de diversas localidades (Tánger, Cádiz, Málaga, Gibraltar, Nápoles, Suecia, Noruega, Helgoland y de otras sin indicación precisa) probablemente de algólogos de la época (Cabrera, Schousboe, C. Agardh, Haenseler, Prolongo, Magnus y otros que ofrecen dudas, como Correns, Jaenicke y Müller).

En este primer trabajo tan sólo publicamos aquellos pliegos en que coincidía el binomen antiguo con el material identificado. Son 148, lo que representa 96 táxones (60 Rhodophyceae, 27 Phaeophyceae y 9 Chlorophyceae).

Por otra parte, hay que destacar el pliego no 33 de Cádiz (Fucus floresius Clemente), al que consideramos como neótipo de Halymenia floresia (Clemente) C. Agardh, y el nº 34 también de esta localidad, nos avala la prioridad de Zonaria tournefortii (Lamouroux) Montagne sobre Z. flava (Clemente) C. Agardh, que es lo que dice Nizamuddin, 1981: 32.

Palabras clave. Sociedad Malagueña de Ciencias, herbario, algas.

ABSTRACT. Through Bellon's work (1940) we found in the Oceanographic Laboratorie of Malaga, a part of the herbarium of the Scientific Society of Malaga (HSMC).In this herbarium, 150 years old material was found from different locations (Tánger, Cádiz, Málaga, Gibraltar, Nápoles, Suecia, Noruega, Helgoland, as well as others not well defined), probably from algae specialists of that time (Cabrera, Schousboe, C. Agardh, Haenseler, Prolongo, Magnus, as well as others doubtful ones e. g. Correns, Jaenicke and Muller).

Out of 242, 148 individuals have been examined, representing 96 taxons $(60$ Rhodophyceae , 27 Phaeophyceae, and 9 Chlorophyceae ). The remaining material, either it was mislabeled, or too damaged, since it was clear that others hands had passed through it and some accident may have occured.

In the other hand, the folio $\mathrm{n}^{\mathrm{0}} 33$ of Cádiz (Fucus floresius Clemente) is considered as a neotype of Halymenia floresia (Clemente) C. Agardh and folio no34, also from this town, enhance the priority of Zonaria tournefortii (Lamouroux) Montagne over $Z$. flava (Clemente) C. Agardh, as was indicated by Nizamuddin 1981: 32.

Key words. Malaga's Scientific Society, herbarium, seaweeds.

\section{INTRODUCCIÓN}

La Sociedad Malagueña de Ciencias, fue una entidad que fundaron Pablo Prolongo y García (1806-1885) y Domingo de Orueta Aguirre (1833-1895) en 1872 
y que en su época dispuso del herbario de Haenseler (1766-1841) y parte del de Cabrera (1762-1827), con pliegos de Clemente (1777-1827), Schousboe (17661832), Carlos Agardh (1785-1859) y del herbario del mismo fundador (Laza, 1945: 220 y Carrillo et al., 1984: 21a, 23 y 27). (Láminas I y II).

Los avatares por los que han pasado estos pliegos y principalmente los de algas, y aún ¿cuando se formó esta colección ? nos los detalla Bellón (1940: 2), no obstante reseñaremos que el susodicho material al que alude la bibliografía es tan sólo al de Clemente, 29 pliegos de los que hemos podido comprobar 26 en sus propios materiales originales (Conde, 1981: 102).

Tenemos pruebas por observación directa en Granada de la existencia de los de «Clemente « ya que existe coincidencia en lo que dice Bellón,1940: 3 y siguientes, con lo observado, aparte de la etiqueta de revisión que data de 1938 y el poner en ellas «Herbarium Societatis Malacitanae Scientiarum «, pero sin embargo las de Málaga 89 a 137 (Lám. III, fig. 4) no son del anterior autor, ya que en ellas junto con las otras de las diferentes localidades figurarán separadas por el «papel «(ficha del Instituto Español de Oceanografía. Sección de Biología, (Lám. III,fig. 6) donde iría el estudio de las dimensiones de una determinada especie de pez) en donde con letra de Bellón se observa la pertenencia a la Sociedad y al final la fecha de 1939, con lo que se nos da una muestra más de que los pliegos clementinos se separaron de los otros, concluyéndose su estudio en Julio de 1938 (Bellón, o.c.) y estos posteriores quedaron en el tintero.

Está claro que ambos autores (Bellón,1940 y Laza, 1942) decidieron estudiar cada uno, la parte en la que estaban especializados, de forma que al no existir por aquel entonces lugar « suficiente» donde ubicar aquellos pliegos, éstos, pasaron (la mayoría de Fanerógamas) al departamento de Botánica de la Facultad de Farmacia de la Universidad de Granada; es por lo que juntamente con aquellos pasaron otros también de Málaga, números 58 a 88 (mezclados con éstos, se encuentran algunos de los 29 de Clemente) de distinta época y otros de Bandorán, Cork Harbour e Ireland.

Bellón (o. c.) nos relatará más tarde que en el mismo herbario y casi terminando el estudio de las de Clemente, descubrió otros, pero ya no hizo ninguna investigación posterior, referente a este material.

D. Luis Bellón perteneció al Laboratorio Oceanográfico de Málaga desde los años de 1935 a 1954; publicó otros trabajos sobre algas («Flora Boetica», Flora del Mar de Baleares», ...) (Navarro, 1955: 19) y allí en el Oceanográfico de Málaga quedaron estos pliegos, amontonados con la idea de que algún día los pudiera estudiar. Posteriormente y con motivo del inicio de mi tesis doctoral en 1975 y revisando la abundante biblioteca de Bellón en el oceanográfico malagueño , descubro estos pliegos y con permiso del antes director de aquella entidad D. Natalio Cano, estos ejemplares pasan a mi poder quedando depositados en el Departamento de Botánica de Málaga.

Estos son los 242 pliegos (tan sólo se han podido estudiar unos pocos, generalmente aquellos cuya identificación era correcta, aunque en la etiqueta se presenten con nomenclatura antigua) con la numeración, procedencia geográfica y probablemente por quién los «clasificó», según L. Bellón, 1939 (ya que así lo pone con un papel,que dice: «Herbario de la Sociedad Malagueña de Ciencias «y que precede a cada bloque o paquetes de pliegos y separados unos grupos de otros mediante un cordelito que los reunía).(Lámina III).

A. Tánger $\mathrm{n}^{\circ} 1$ a 29 («clasificadas», probablemente, por Schousboe y enviadas por éste a Haenseler).

B. Cádiz nº 30 a 57 («clasificadas», algunas cédulas están trastrocadas. Comunicadas por Cabrera a Haenseler). 
C. Málaga (presuponemos $\mathrm{n}^{\mathrm{o}} 58$ a 88 y que están en el herbario de la Universidad de Granada).

D. Málaga no 89 a 137.(«clasificadas», la mayoría de las cédulas son de letra de $¿$ Haenseler?.Algunas trastrocadas, no correspondiente a los ejemplares contenidos en los pliegos. En la mayoría de los pliegos no existen localidades, salvo muy pocas que ponen el Peñón del Cuervo, cerca de Málaga. Normalmente ponen: «passim», «communis», «frequens», ...).

E. Gibraltar $n^{\circ} 138$ a 141 («clasificadas».El tipo de letra es el de la Sra. inglesa que dice Bellón y similar al de las algas de Cork y Málaga, en Granada).

F. Sin indicación de localidad no142 a 167. (¿Inservibles?. Sin fechas, ni no de etiquetas, salvo rarísimas excepciones, sin localidad. Muchas veces la letra de la cédula es desconocida, otras veces, los ejemplares no están pegados sobre el papel y en estos casos parece como si posteriormente se hubiera añadido una pequeña etiqueta con el nombre, puesto por la Señora inglesa).

G. Nápoles nº 168 y 169 (Están «clasificadas»).

H. Suecia no 170 a 181 («clasificadas». Enviadas a Haenseler ?).

I. Helgoland no 182 a 208 («clasificadas»).

J. Extranjeras sin indicación precisa de localidad nํㅜㄹ. 209 a 220.(«clasificadas»).

K. Noruega 221 a 223 («clasificadas»). en Granada.)

L. Bandorán, Cork Harbour e Ireland (presuponemos nº 224 a 242 y que están

También mezclados con estas colecciones de algas existen pliegos desde los $\mathbf{n}^{\circ}$ 243 a 258 que son de Celentéreos, Briozoos, Zosteráceas, Esponjas, Pennátulas, etc. y otros 79, probáblemente de Málaga, de la época de Haenseler, pero que no ponen ni fecha, ni localidad, ni identificación, en una palabra « Inservibles «, según comentarios de Bellón.

Este material fue clasificado y comunicado por Schousboe, Cabrera, Agardh («por entonces envió Agardh a Haenseler ejemplares dedicados de sus publicaciones (Algarum Decas... )que se conservan en la Sociedad Malagueña de Ciencias» Bellón,1942: 21), Prolongo, Magnus, algunos desconocidos pero incluso de 1831; hay otros en que la duda permanece si son de Cabrera?, Haenseler?, Agardh?, Magnus?, Correns?, Jaenicke?, Müller?.

Por último hay que hacer constar y también en el Laboratorio Oceanográfico de Málaga, que encontramos en papeles sueltos (Navarro, 1955: 18), a manera de fichas y escritas por Bellón, todas y cada una de las copias sobre las cédulas que debieran de corresponder a cada pliego, donde se recoge el texto de las fichas originales (que están en sus pliegos) con comentarios y principalmente y en lápiz rojo, de quien es la letra original de lo que figurase escrito en pliegos o en fichas.

La debacle y trastroques de fichas, cierto desorden en suma, (ya que no pasó completamente desapercibido este herbario y la sinonimia de nombres posteriores que aparecen a veces en los mismos pliegos, sea debida a la misma persona, señora inglesa que dice Bellón), sinonimias antiguas, endurecimiento y bastantes ejemplares y etiquetas estropeadas por más del siglo y medio de algunos ejemplares que llevaría este material fuera de su ambiente, hacen desistir y desanimar, siendo imposible la identificación específica en múltiples ejemplares; pero inducido por las frases de Bellón, o.c. y Seoane,1969: 213, relativas a la escasez de colecciones fícicas y antiguas en nuestra patria, nos alentaron para dar a la luz este material, después de protegerlo, cambiarlo de carpetas (las anteriores de papel de estraza y gran parte carcomidas) y en éstas darles el número original del pliego del HSMC y poner localidad, para así evitar pérdidas y facilitar la consulta. 


\section{TRANSCRIPCIÓN DE LAS CÉDULAS}

A continuación se exponen ordenadas según la agrupación, en bloques de las distintas localidades de donde proceden, haciendo una transcripción de las fichas o etiquetas de los diferentes pliegos; actualizando las sinonimias y viendo si aquellas coincidían con nuestra identificación. Irá también, y al principio, el número de pliego de herbario de la ficoteca del Departamento de Biología Vegetal de Málaga (MGC PHYC) o con el de Granada (GR); ya que como exponíamos en la introducción algunos los observamos en la Facultad de Farmacia de Granada , y los otros , la gran mayoría quedan a disposición en el herbario de Málaga.

Los símbolos tipográficos y abreviaturas sobre las transcripciones de las etiquetas, han sido los empleados por otros autores (Bellón, 1940; Burdet, Charpin y Jacquemoud, 1981: 544-545; Barra y López-González, 1984: 346-347 y Bueno, Gutiérrez-Bustillo y Navarro, 1985: 349) y que parecen estandarizados.

A. Algas de Tánger:

3. Hypnea musciformis (Wulfen) Lamouroux, 1813

MGC PHYC: 1937.

Haenseler].

a. $\square$ N 2526 / Fucus acerosus Schousb. / sp. n. // e mari tingitano a D. Schousb. [m.

8. Ulva lactuca L. f. cribosa J. Ag. 1883

MGC PHYC: 1938.

a. $\square$ N 2661 / Ulva cribrata Schousb / U. agara Gmel. //e mari tingitano [m. Haenseler].

(Lám. I, Fig. 1).

Observaciones: El nombre correcto sería cribaria.

9 y 10. Gracilaria verrucosa (Hudson ) Papenfuss, 1950

MGC PHYC: 1939.

a. $\square$ N. s/n. / Fucus confervoides ? / var?. // a D. Schousb. [m. Haenseler].

b. $\square$ N. 2547./Fucus circinalis, Schousb./ sp. n. // e mari tingitano [m. Haenseler] que corresponde a $\boldsymbol{G}$. compressa.

12. Ceramium ciliatum (J. Ellis) Ducluz., 1809

MGC PHYC: 1940.

a. $\square$ N. 2681 [tachado el 7 que había en lugar del 6 y enmendado] / Conferva pilosa Roth // e mari tingitano [m. Haenseler]

13. Calliblepharis ciliata (Huds.) Kützing, 1843

MGC PHYC: 1941.

a. $\square$ N. 17 [tinta oscura; letra de Haenseler] / Cillepora [?] // e mari tingitano [m. Schousboe].

Observaciones: Al dorso hay escritas unas palabras eslavas [«...gzudt lougrze»]. Esta pequeña cédula (aproximadamente $60 \times 48 \mathrm{~mm}$ ) está escrita en el reverso de un trozo de nota.

15. Chylocladia verticillata (Lightf.) Bliding, 1928

MGC PHYC: 1942.

a. N. 2616 / Fucus salicornia Schousb. // e mari tingitano [m. Haenseler]

Rev.: Fucus salicornia Schousb. // e mari tingitano [m. Schousboe]. (Ver Lám. I, Fig. 6).

19. Dictyopteris polypodioides Lamouroux, 1809

MGC PHYC: 1943.

a. $\square$ N. 2608 / Fucus [m.i.: Fucus está tachada] polypodioides Desf. / Haliseris polypodioides Ag. [m.i.: ¿Señora inglesa?] // a D. Schousb. / e mari tingitano [m. Haenseler, esta etiqueta, al igual que las otras de este autor, mide 127 x $95 \mathrm{~mm}$.]. 
21. Halurus equisetifolius (Lightf.) Kütz., 1843

MGC PHYC: 1944.

a. $\square 2603$ /Fucus pilosus Schousb. / sp.n. // e mari tingitano [m. Haenseler].

24. Caulerpa racemosa (Forsskäl) J. Ag., 1872

MGC PHYC: 1945

Haenseler].

a. $\square$ N. 2611 / Fucus racemosus Schousb. / sp. n. // e mari tingitano et gaditano [m.

26. Liagora viscida (Forsskäl) C.Ag., 1822

MGC PHYC: 1946.

a. N. 2643 [m. Haenseler].

Rev.: Fucus viscidus e mari tingitano [m. Schousb.].

28. Halidrys siliquosa (L.) Lyngb., 1819

MGC PHYC: 1947

a. $\square$ N. 2621 / Fucus siliquosus L. // a D.Schousb. [m. Haenseler].

29 ter. Membranoptera alata (Huds.) Stackh., 1809

MGC PHYC: 1948.

a. N. 2528 / Fucus alatus / Delesseria alata [m. i., Señora inglesa] // a D.Schousb. [m. Haenseler, el texto va en el mismo pliego en el que va pegado el ejemplar y que en este caso mide $159 \times 113 \mathrm{~mm}]$.

B. Algas de Cádiz.

33. Halymenia floresia (Clemente) C. Ag., 1822

MGC PHYC: 1949.

a. $\square$ N. 1159 [m.i.: tachado], 1668 [m.i.: tachado] 2568 / Fucus Floresius Clemente.

/ [aparece la descripción del ejemplar según el « ensayo de la vid «p. 312, de Clemente] // in mari gaditano a D. Cabrera [m. Haenseler. Etiqueta destruida a partir de la supuesta «a última» de Cabrera.].

Observaciones: En el pliego aparece escrito $F$. floresius / $\mathrm{Clem}^{\text {te }}[\mathrm{m}$.i.: que no parece de Cabrera]. (Lám. VI). Codomier (1974: 3) que estudia ampliamente el taxon no nos da referencias que existan muestras tipos de la especie en algún herbario, tal y como hiciera por ejemplo con H. trigona (Clemente) C. Ag., es por lo que lo consideramos como posible neótipo.

34. Zonaria tournefortii (Lamouroux) Montagne, 1846

MGC PHYC: 1950

a. $\square$ N. 1151 [m.i. tachado], 1660 [m.i. tachado], 2567 / Fucus flavus L. / F. spongiosus , ramis erectis subquinquangu-/laribus laciniato-dentatis areolatis nitidis / . L. supl. 452.Pers. Syst. Veg. p.1010// a D.Cabrera in mari gaditano [m. Haenseler]. (Lám. I, fig. 2).

Observaciones: Presuponemos que esta descripciòn sea la que figure en Linnaeus, 1781.- Supplementum plantarum. Consultando Nizamuddin ,1981: 32, observamos que da la prioridad a Z. flava (Basionym: Fucus flavus Clemente,1804: ?; 1807: 310, non Linnaeus 1781:452; 1792: 390) - está claro que la descripción de la etiqueta de $F$.flavus L. no corresponde a Zonaria -.Viendo la bibliografía del trabajo de Nizamuddin (o.c.) en que nos refiere a Clemente 1804, en esta última reseña dice: (cf. De Candolle,1818: 34).Hemos observado el trabajo de De Candolle (o.c.) en un antigüo ejemplar de la Sociedad Malagueña de Ciencias y efectivamente viene el trabajo de Clemente de 1804 - que es el de 1807-pero dice: «traduit de l'espagnol por L. M. Caumels» y una nota escrita en el màrgen inferior del libro,en làpiz , que creemos letra de Prolongo y que dice: «(¡Clemente!, está equivocado: es de 1814) «, así es , ya que en Tellez y Alonso, 1952: 31, en la biografía sobre Clemente se habla de la traducción al francés del «ensayo» por el marqués de Caumels en 1814 y en Colmeiro 1858: 86 , tambièn trata de la citada traducciòn, aunque no se diga por quien. Luego el F. tournefortii 
de Lamouroux 1805 es prioritario al F. flavus de Clemente 1807 y por lo tanto la Z.tournefortii, (Lám. IV, fig.1) en contra de lo que dice Nizamuddin (o.c) por lo que la revalidamos.

\section{Liagora distenta (G. Mertens ex Roth) C. Agardh, 1822}

MGC PHYC: 1951

a. $\square$ N. 547 [m.i. tachado], 1184 [m.i. tachado], 1691 [m.i. tachado], ...59 [ya que las primeras cifras no existían por estar el papel roto] / Fucus canalicularioides Clemente / [a continuación aparece la descripción del ejemplar según: «Clemente variedades de la vid, p.318] / Fucus distentus Mertens Roth Cat. III. // circa Marbella, / in mari gaditano a D. Cabrera [m. Haenseler].

36. Taonia atomaria (Woodw.) J. Ag., 1848

MGC PHYC: 1952.

a. $\square$ N. 1205 [m.i. tachado], 1721 [m.i. tachado], 2664 / Ulva fastigiata Clem $^{\text {te }} /$ difert ab praecedenti [tachada esta palabra interlineadas las dos siguientes] Ulva dichotoma fronde stipitata crassiuscula / spureo-pellucida, valde irregulariter dichotoma / subdentata // in mari gaditano a D. Cabrera [m. Haenseler].

b. [ejemplar pegado sobre el papel, más estrecho que el de la etiqueta anterior] / Ulva fastigiata / Clemente [m.i.] // Dictyota / dichotoma / [lo que no es, m.i.: ¿Señora inglesa?]

Observaciones: En el mismo pliego aparece material suelto de Dictyota dichotoma.

39. Phyllophora pseudoceranoides (Gmelin) Newrot et Taylor,1971 (= Ph. membranifolia (Goodn. \& Woodw.) J.Ag.)

MGC PHYC: 1953.

a. $\square$ N. 1133 [m.i. tachado], 1641 [m.i. tachado], 2585 / Fucus membranifolius / F. caule tereti ramoso; apicibus in folia enervia / sub-biloba expansis; tuberculis seminiferis / e cauler pedunculatis. Linn.trans. p. 120 t. 16 f / Turner synops. p. 25 / F. palmeta fl. Dan. t. 827 // in mari gaditano a D. Cabrera [m. Haenseler].

Observaciones: Hay que hacer notar que Seoane, 1965, no la cita; aunque si la diera Clemente, 1807: 308 sub Fucus membranifolius L.

\section{Ectocarpus fasciculatus? Harvey, 1841}

MGC PHYC: 1954.

a. N. 1216 [m.i. tachado], 2691 [m. ¿Haenseler?] / Conferva species indes- / cripta / in litore Gadisen / si / [m. Cabrera. La última sílaba está superpuesta, interlineada].(Lám. I, fig. 8). IV, fig. 3).

Observaciones: En un lateral Ectocarpus fasciculatus ?[m.i. ¿Señora inglesa?] (Lám.

42. Phyllophora crispa (Hudson) Dixon, 1964

$(=$ Ph. rubens $(\mathrm{L}$.$) Grev. =$ Ph. epiphylla $($ Muller $)$ Batters $)$

MGC PHYC: 1955.

a. $\square$ N. 2614 / Fucus rubens L. / var. // a D. Cabrera e mari gaditano [m. Haenseler].

45. Fucus vesiculosus L., 1753

MGC PHYC: 1956.

a. $\square$ N. 532 [m.i. tachado], 1148 [m.i. tachado], 1657 [m.i. tachado], 2640 / Fucus vesiculosus / [la etiqueta está apolillada y tiene múltiples agujeros, , implicando gran parte del texto (donde figura descripción y Herb. Linn. Turner Syn. t. 1 p. 117)] // a D. Cabrera in mari gaditano [m. Haenseler. Aunque se interpreta bien este texto, está muy apolillado]

46. Rytiphloea tinctoria (Clemente) C.Agardh, 1817

MGC PHYC: 1957.

a. $\square$ N. 1173 [m.i. tachado], 1681 [m.i. tachado], 2633 / Fucus tinctorius Clem ${ }^{\text {te }} /$ [la etiqueta está muy apolillada que dificulta la descripción del taxon según: Clemente Ensayo de la vid p.317] // in mari gaditano a D. Cabrera [m. Haenseler]. 
47. Enteromorpha compressa (L.) Grev., 1830

MGC PHYC: 1958.

a. $\square$ N. 555 [m.i. tachado], 1203 [m.i. tachado], 1715 [m.i. tachado], $2661 /$ Ulva compressa L. / Agardh 1. c. p. 23 // passim in mari gaditano a D. Cabrera [m. Haenseler].

50. Enteromorpha ramulosa (Sm.) Hook., 1833

MGC PHYC: 1959

a. $\square$ N. 2685 / Conferva longissima Desf. // e mari gaditano [m. Haenseler].

51. Dictyota dichotoma (Huds.) Lamouroux, 1809

MGC PHYC: 1960.

a. $\square$ N. 1204 [m.i. tachado], 1720 [m.i. tachado], 2663 / Ulva dichotoma vs. // in mari gaditano a D. Cabrera [m. Haenseler].

b. Ulva dichotoma Clementis vars. [m. Cabrera] (Lám. I, fig. 7).

52. Gastroclonium ovatum (Huds.) Papenfuss, 1944

MGC PHYC: 1961.

a. $\square$ N. 1136 [m.i. tachado], 1644 / Fucus ovalis / F. fronde filiformi subcompressa vagè ramosâ; / ramis uniformibus; foliis ovali-oblonguis car- / nosis integerrimis, superioribus confertis. / Linn. trans. iii p. 116. Turn. 1. c. p.31.//F. uvarius Sp. [m. Haenseler, tachado Sp.] Syst. veget. ed. Pers. Esp. p.153 t.78, f.1 // in mare gaditano a D. Cabrera .

Observaciones: Señalemos que Seoane, 1965, no la cita para Cádiz, pero sí Clemente, 1807: 308, sub Fucus ovalis Hudson.

\section{6 y 57. Gracilaria foliifera (Forsskäl) Börg.,1932}

MGC PHYC: 1962.

a. $\square$ N. 535 [m.i. tachado], 1156 [m.i. tachado], 2586 / Fucus multipartitus Cl. / F. fronde plana enervi coriacea laciniato- / subpalmata margine subciliata, laciniis / multipartitis, ciliis patentibus, tuberculis / inmersis hinc concavis. Clemente / var. de la vid. p. $311 / /$ in mari gaditano a D. Cabrera [m. Haenseler].

b. $\square$ N. 1157 [m.i. tachado], 1166 m.i. tachado], 2587 / Fucus multipartitus Clem $^{\text {te }}$ var. $/ /$ in mare gaditano [m. Haenseler].

C. Algas de Málaga en Granada:

Los números 58 a 88 son de Málaga y estaban incluidos en el Herbario de la Facultad de Farmacia de Granada, en 3 cajas de algas (A-CH, D-N y O-Z). En dichas cajas también estaban los pliegos de Clemente y que publicara Bellón (o.c.).En mi tesis (Conde, 1981) de forma más amplia y posteriormente en Conde, 1984 se citan los ejemplares que pudimos comprobar en sus propios materiales originales.

No hemos podido comprobar in situ (por comparación con los «papeles sueltos a manera de fichas» de Bellón ) todos los ejemplares, pues algunos tuvieron que desaparecer . En las cédulas originales casi siempre no tienen número, la letra parece ser de la Sra. inglesa, que escribe en tinta roja y que Bellón comenta en su trabajo (Bellón, 1940: 4). Luego, daremos relación tan sólo de los que vimos en Granada, sin la confirmación definitiva de su identificación y con la numeración que tenemos, de las fichas de Bellón, pero que no es la que corresponde a la que tiene en Granada, ya que aquí en un mismo pliego existe material de distintos recolectores, aunque estuviesen separados; estos recolectores son: Dieguez, Cobos, Hurtado, $\ldots$ aunque de años posteriores $(1941,1946, \ldots)$ a lo que es motivo este trabajo.

59. Laurencia pinnatifida (Huds.) Lamour., 1813

GR.: 467

a. s/n. / Laurencia pinnatifida / Málaga. [m.i.: ¿Señora inglesa?]

Observaciones: Hay 4 folios, en uno de ellos existen dos ejemplares, uno, es éste.

60. Gracilaria verrucosa (Huds.) Papenfuss, 1950

GR.: 539 

inglesa?].

a. s/n. /Gracilaria / confervoides / Málaga / Febry. 26 th. / (dreged).[m.i.: ¿Señora

Observaciones: En uno de los 4 folios que hay, uno de ellos es de Clemente.

61. Cystoseira nodicaulis (With.) Roberts, 1967

GR.: $380 \mathrm{~b}$.

a. s/n. / Cystoseira / granulata / Málaga / March 3 [m.i. ¿Señora inglesa?].

Observaciones: Aunque sólo existe un folio de $C$. nodicaulis, también hay otros de $C$. tamariscifolia, C. stricta y C. spinosa.

62. Halopteris filicina (Grateloup) Kütz., 1843

GR.: 332.

a. s/n. / Sphacelaria / filicina / Málaga / Febry. 26 th. [m.i. ¿Señora inglesa?].

Observaciones: Aquí existen 3 folios, en cada uno de ellos hay dos ejemplares; en todos van como Halopteris o Stypocaulon scoparia. Tan sólo hay uno que figura como Sphacelaria filicina. No obstante, con lápiz, pone después: S. scoparia. Hay decir también que uno de los 6 ejemplares sería el de Clemente.

63. Cystoseira tamariscifolia (Huds.) Papenfuss, 1950

GR.: $380 \mathrm{~b}$.

a. s/n. / Cystoseira / ericoides / Málaga / Febry [m.i. ¿Señora inglesa?].

Observaciones: Aquí hay tres folios de Cystoseira ericoides, uno sería de Clemente.

Ver también número 61.

64. Petalonia fascia (O.F. Müller) Kuntze, 1891

GR.: $347 b$

a. s/n. / Laminaria / debilis / Málaga / Febry. 26th. / (growing).[m.i. ¿Señora inglesa?] Observaciones: Hay dos folios, el otro correspondería al número 86.

65. Pterosiphonia complanata (Clemente) Falkenb., 1901

GR.: 477

a. N. 15.a. / Rytiphlaea / complanata. / Málaga / Febry [m.i. ¿Señora inglesa?]

Observaciones: Hay dos folios, el otro corresponde al número 77.

66. Gymnogongrus crenulatus (Turner) J. Ag., 1851

GR.: 596

a. s/n. / Chondrus / novergicus. / Málaga [m.i. ¿Señora inglesa?]

Observaciones: Hay 5 folios, en uno de ellos hay dos ejemplares, en donde está el nuestro. Otro folio tiene el ejemplar de Clemente y otro de los folios tiene tres ejemplares de 1941. Ver Conde, 1981: 225,226 y 228,229.

68. Gracilaria foliifera (Forsskäl) Börg., 1932

GR.: 538 .

a. s/n. / Gracilaria / multipartita / Málaga / February 1851 [m.i. ¿Señora inglesa?]

Observaciones: Hay tres folios, uno de ellos sería el de Clemente.

69. Laurencia hybrida (A. DC.)Lenorm. ex Duby, 1830

GR.: 473b.

a. N. 23. / Laurencia / cespitosa / Málaga / February [m.i. ¿Señora inglesa?, tan sólo hay un folio]

72. Schizymenia esbyi (Chauv. ex Duby) J. Ag., 1851

GR.: 588

a. s/n. / Iridea / edulis / Málaga/ [m.i. ¿Señora inglesa?]

Observaciones: En este pliego del herbario de GR., existen varios folios: de Dieguez (abril,1946), Cobos (22 / VI / 41), y otros.Uno de ellos sería el de Clemente. (Conde, 1981: 214);no obstante ver Pérez-Cirera,Cremades y Bárbara (1989: 124, 131 y 133) en que consideran que pudiera ser Grateloupia lanceola (J.Ag.) J. Ag. emend. Ardré \& Gayral, el material de Hurtado de Mendoza de Málaga del 1948. 
73. Gigartina pistillata (S. G. Gmelin) Stackh., 1809

GR.: 603

a. s/n. / Gigartina / pistillata / Málaga [m.i. ¿Señora inglesa?]

Observaciones: Hay 4 folios,uno sería el de Clemente.

74. Pterothamnion plumula (J. Ellis) Nägeli, 1855

GR.: 432 un folio]

a. s/n. / Callithamnion / plumula. / Málaga / March.[m.i. ¿Señora inglesa?. Hay tan sólo

Observaciones: Rev.: «Growing on the ... [2 palabras] stones along the mole».

76. Bryopsis plumosa (Huds.) C. Ag., 1822

GR.: 189. inglesa?].

a. s/n. / Bryopsis / plumosa / Málaga / March. 2 / Growing on rocks [m.i. ¿Señora

77. Pterosiphonia complanata (Clemente) Falkenb., 1901

GR.: 477.

a. s/n / Rytiphlea / Málaga / March 16th. [m.i. ¿Señora inglesa?]

Observaciones: Ver también número 65.

78. Callithamnion tetragonum (With.) S. F. Gray, 1821

GR.: 422.

a. s/n / Callithamnion / tetragonum / Málaga [m.i. ¿Señora inglesa?]

80. Gigartina acicularis (Roth) Lamouroux, 1813

GR.: 605.

a. s/n / Gigartina / acicularis / Málaga / March 3. [m.i. ¿Señora inglesa?]

Observaciones: Hay cuatro folios, uno de ellos es el de Clemente.

82. Dictyopteris polypodioides Lamouroux, 1809

GR.: 360

inglesa?]

a. N. 8.11 [tachado este número] Halyseris / polypodioides. /Málaga [m.i. ¿Señora

Observaciones: Hay dos folios, en uno de ellos, a su vez, existen dos ejemplares.

83. Calliblepharis jubata (Good. et Woodw.) Kütz., 1843

GR.: 550.

a. s/n / Rhodymenia / jubata / Málaga / Febry [m.i. ¿Señora inglesa?]

Observaciones: Hay tres folios, uno es el de Clemente; en otro hay dos ejemplares.

86. Petalonia fascia (O.F. Müller) Kuntze, 1891

GR.: 347b.

a. s/n / Laminaria / debilis Ag. / Málaga / March 16 th [m.i. ¿Señora inglesa?]

Observaciones: Ver también número 64.

88. Grateloupia filicina (Lamouroux) C. Ag., 1822

GR.: 578 .

a. s/n / Grateloupa [le falta la i]/ filicina . / Málaga [m.i. ¿Señora inglesa?]

D. Algas de Málaga.

91. Colpomenia sinuosa (Mertens ex Roth) Derbès et Solier,1851

MGC PHYC: 1963

a. $\square$ N. 554 [m.i. tachado] 1208 [m.i. tachado] 1423 [m.i. tachado] 2669 / Ulva sinuosa Mertens / U. cavernosa Forsk.? / U. vesicaria Schousb // communis apud nos [m. Haenseler]. 
91 bis. Ulva rigida C. Ag., 1824

MGC PHYC: 1964.

a. $\square$ N. 2668 / Ulva lactuca / var // communis [m. Haenseler].

92. Laurentia obtusa (Hudson) Lamouroux, 1813

MGC PHYC: 1965

a. s/n. / Fucus obtusus Hudson / Vs.B. apd. nos / [m. ¿Cabrera?].

93. Peyssonnelia squamaria (S. G. Gmelin) Dcne., 1841

MGC PHYC: 1966

a. $\square$ N. 526 [m.i. tachado] 1135 [m.i. tachado] 16...0 [m.i. tachado y apolillado] 2624

/ Fucus squamarius Gmelin // communis [m. Haenseler].

95. Gigartina teedii (Roth) Lamour., 1813

MGC PHYC: 1967

a. $\square$ N. 2632 / Fucus Theedii Mertens / Roth Cat. bot. 3.p.108// passim [m. Haenseler].

96. Cystoseira tamariscifolia (Hudson) Papenfuss, 1950

MGC PHYC: 1968.

a. $\square$ N. 530 [m.i. tachado], 1145 [m.i. tachado], 1654 [m.i. tachado], 2631 / Fucus tamariscifolius Hudson / [a continuación la descripción del taxon según Turner. Syn. t.1.p.88] / Fucus ericoides Linn. Trans. iii p.130 // communissimus peremnis [m. Haenseler].

96 bis. Corallina officinalis L. 1758

MGC PHYC: 1969.

a. $\square$ N. 8 [m.i. tachado] 18 / Corallina officinalis L. // communis [m. Haenseler]

Observaciones: También existe material de Amphiroa rigida Lamour., 1816.

98. Pterosiphonia complanata (Clemente) Falkenb.,1901

MGC PHYC: 1970.

a. $\square$ N. 2645 / Fucus complanatus Clem. // in Peñón del Cuervo [m. Haenseler].

99 y 100. Gracilaria foliifera (Försskal) Börgesen, 1932

MGC PHYC: 1971

a. $\square$ N. 2589 / Fucus multipartitus Clemte / varietas // in mari malacitano et gaditano

[m. Haenseler].

b. $\square$ N. 2590 / Fucus multipartitus Clem. / var // passim in mari malacitano [m.

Haenseler].

101. Codium vermilara (Olivi) Chiaje, 1829.

MGC PHYC: 1972.

a. $\square$ N. 548 [m.i. tachado], 1185 [m.i. tachado], 1635 [m.i. tachado], 2634 / Fucus tomentosus Huds. / [descripción según Flor. angl. p.584 / Turner p.300] / Lamarkia vermillaris Olivi [m.i.] / Codium tomentosum [m.i. Señora inglesa] // communis [m. Haenseler].

102. Padina pavonica (L.) Lamouroux, 1816

MGC PHYC: 1973.

a. $\square$ N. 525 [m.i. tachado], 1134 [m.i. tachado], $16 / 2600$ [m.i. tachado: ¿Señora inglesa?], / Fucus pavonius L. Sp. pl. 2. p.1630. / Ulva pavonia [m.i. tachado ¿Señora inglesa?]L. Syst. Veg. / Padina pavonia (Adams.) [m. Señora inglesa] // communis apud nos [m. Haenseler].

103. Halopitys incurvus (Huds.) Batters, 1902 MGC PHYC: 1974.

a. $\square$ N. 540 [m.i. tachado], 1191 [m.i. tachado], 1701 [m.i. tachado], 2604 / Fucus pinastroides [descripción del taxon según: Gmelin p.127 t. 11 f. 1 / Turner Syn. p..346] / Rytiphlea pinastroides / Ag./ [m. Señora inglesa] // communissimus [m. Haenseler]. 
105. Laurencia pinnatifida (Huds.) Lamouroux, 1813

MGC PHYC: 1975.

a. $\square$ N. 541 [m.i. tachado], 1170 [m.i. tachado], 1677 [m.i. tachado], 2605 / Fucus pinnatifidus Turn. [descripción del taxon según: Turner. Syn. p. 267]// apud nos ad rupes maris communis [m. Haenseler].

106. Dictyota dichotoma (Huds.) Lamouroux, 1809

MGC PHYC: 1976.

a. $\square$ N. 557 [m.i. tachado], 1203 [m.i. tachado], 1719 [m.i. tachado], 2662 / Ulva dichotoma Huds // in utrisque maribus mediterraneo oceanoque [m. Haenseler].

Observaciones: Entremezclado existe material de Taonia atomaria.

107. Jania rubens (L.) Lamouroux, 1812

MGC PHYC: 1977

a. $\square$ N. 19 [primero se escribió el 9, después, con letra más oscura, se añadió el 1] / Corallina [m.i. tachado] rubens L. / Jania rubens Lamx. / Hist. pol. p. 27 [estas dos últimas líneas con letra posterior y tinta más oscura]/varietates diversae // communis [m. Haenseler].

111. Pterocladia capillacea (Gmel. ) Born. et Thur., 1876 MGC PHYC: 1978.

a. $\square$ N. 1176 [m.i. tachado], 1684 [m.i. tachado], 2549 / Fucus corneus vs. capillaceus / F. fronde tenui; pinnis apicen versus / confertis sub-cetaceis erectiusculis / Turner p. 273 / [m. Haenseler] / Gelidium corneum Harvey [m. Señora inglesa] // communis [m. Haenseler].

115. Gigartina pistillata (S.G. Gmelin) Stackh., 1809

MGC PHYC: 1827.

a. $\square$ N. 543 [m.i. tachado], 1179 [m.i. tachado], 1687 [m.i. tachado], 2572 / Fucus gigartinus L. sp. pl. // ... diversa specie habet D. Schousboe // [En el margen izquierdo aparece roto el papel,con lo que no se lee toda la frase] //communis [m. Haenseler].

Observaciones: De este pliego ya dimos noticias en Flores-Moya, Vera-González y Conde, 1989: 200.

116. Schizymenia dubyi (Chauvin ex Duby) J. Ag., 1851

MGC PHYC: 1979.

a. $\square$ N. [?] 1155 [m.i. tachado], 1664 [m.i. tachado], 2560 / Fucus edulis / [El ejemplar no está suelto como en la mayoría de los pliegos anteriores, aquí figura pegado a un papel muy destrozado con recuadro hecho con tiralíneas (tinta roja muy decolorada), como los que hiciera Clemente que se hallaban en Granada. La etiqueta está adherida al ejemplar y en ella figura la descripciòn del taxon segùn: «Turner Syn. t.1.p. 180] / F. dulcis Gmelin // in petreis porti malacitani communis [m. Haenseler].(Lám.I, fig. 3 y Lám. IV, fig.2).

117. Gelidium latifolium (Grev.) Born. et Thur., 1876.

MGC PHYC: 1980.

a. $\square$ N. 1175 [m.i. tachado], 16 ... [m.i. tachado], 2548 / Fucus corneus vs. Turner. / Gelidium corneum? (Harvey) / varietas [m.i. Señora inglesa] / varietas haec et sequentes a D. Schousboe pro varietates / F. abrotanifolii L. habentur // communis [m. Haenseler].

124. Ceramium ciliatum (J. Ellis) Ducluz., 1809 MGC PHYC: 1981

a. $\square$ N. 2673 / Conferva ciliata Roth. // vulgaris in mare prope Torremolinos / et Peñón del Cuervo [m. Haenseler].

128. Pterothamnion plumula (J. Ellis) Nägeli, 1855

MGC PHYC: 1982.

a. $\square$ N. 2682 / Conferva [m.i. Señora inglesa, con trazo fino] plumula Roth. / Callithamnion [m.i. Señora inglesa] // in portu malacitano [m. Haenseler]. 
129. Gracilaria foliifera (Försskal) Börg., 1932

MGC PHYC: 1983.

a. $\square$ N. 1158 [m.i. tachado], 1667 [m.i. tachado], 2588 / Fucus [m.i. Señora inglesa, con trazo fino] multipartitus Clemte varietas / Gracilaria [m.i. Señora inglesa] // apud nos in petreis frequens [m. Haenseler].

132. Cladostephus spongiosus (Huds.) C. Ag.,1828 fo. verticillatus (Light.) P.v.R., 1972. MGC PHYC: 1984.

a. $\square$ N. 2680 [aunque el 6 está escrito sobre otro $\mathrm{n}^{\mathrm{o}}$ ] / Conferva myryophyllum [m.i. tachado por la Señora inglesa] Roth / Cat. bot. 3. p. 312. / Cladostephus verticillatus / Agardh [m.i. Señora inglesa] // passim [m. Haenseler, etiqueta muy apolillada].

134. Gigartina acicularis (Roth) Lamouroux, 1813

MGC PHYC: 1985.

a. $\square$ N. 1167 [m.i. tachado], 694 [m.i. tachado], 2545 / Fucus confetvoides v. macrocarpus Clemte // Sed D. Schousboe pro generum diversum habet / F. acicularis Turn. [con tinta más oscura y posterior al resto]/Gigartina acicularis / (Harvey) [m.i. Señora inglesa] // passim [m. Haenseler].

\section{Fucus vesiculosus L.,1753}

MGC PHYC: 1986.

a. $\square$ s/n / Fucus vesiculosus // Málaga [m. Prolongo] (Lám. I, fig. 4 y Lám. V, fig. 4).

Observaciones: Lo que no sabemos es si este material, muy posible recogido por Prolongo, ya que la letra de la cédula sí es, i es arrojado ó no ?; muy probable la primera posibilidad, ya que in situ parece improbable (Conde, 1984: 68).

E. Algas de Gibraltar.

138. Stypocaulon scoparium (L.) Kütz., 1843

MGC PHYC: 1987.

a. s/n. / Sphacelaria / scoparia // Bay of Gibraltar . / Septer. 1850 [m.i. Señora inglesa]. Observaciones: La señora inglesa es a la que alude Bellón (1940: 4).

139. Gelidium latifolium (Grev.) Born. et Thur., 1876

MGC PHYC: 1988.

a. N. 7 / Gelidium corneum / var. latifolium // Gibraltar [m.i. Señora inglesa].

140. Jania rubens (L.) Lamouroux, 1812

MGC PHYC: 1989.

a. N. 17 / Jania / rubens // Catalano / Bay Sept. 1850 [m.i. Señora inglesa].

141. Gelidium sesquipedale (Clemente) Thur. et Thur., 1876

MGC PHYC: 1990.

a. s/n. Gelidium corneum / var. sesquipedale // Gibraltar [m.i. Señora inglesa].

F. Algas clasificadas sin indicación de localidad.

142. Fucus serratus L., 1753

MGC PHYC: 1991.

a. $\square$ N. 1147 [m.i. tachado], 1656 [m.i. tachado], 2619 / Fucus serratus L. / F. fronde lineari dichotoma serrato-dentata; apicibus bifidis planis, esterilibus obtusis tuber-/ culiferis acutis Herb. Linn. Turn. l.c. p. $110 / /$ a D. Cabrera [m. Haenseler].

Observaciones: Nos parece rara la existencia de $F$. serratus en Cádiz y tan sólo conocemos la cita de González-Fragoso (1886: 123).

143. Myriotrichia filiformis Harv., 1848

MGC PHYC: 1992. 
a. s/n / Miriotricha filiformis [m.i.]/ Myriotricha filiformis [m.i. con lápiz].

Observaciones: Sobre Scytosiphon lomentaria.

144. ¿Pylaiea littoralis? (L.) Kjellm., 1872

MGC PHYC: 1993.

a. s/n. / Ectocarpus littoralis [m.i.] Ectocarpus littoralis [m.i. a lápiz].

145. Nemalion helminthoides (Velley in With.) Batters, 1902

MGC PHYC: 1994

a. s/n. / Nemalion multifidum [m.i.].

146. Plocamium cartilagineum (L.) Dixon, 1957

MGC PHYC: 1995.

a. s/n. / Proclamium coccineum [m.i. a lápiz].

147. Phycodris rubens (L.) Batters, 1902

MGC PHYC: 1996.

a. s/n. / Phycodris sinuosa // Delesseria sinuo-/ sa [m.i.ambos binominis a lápiz].

148. Membranoptera alata (Huds.) Stackh., 1809

MGC PHYC: 1997.

a. s/n. / Hypoglossum alatum [m.i. a lápiz].

149, 149 bis y 149 ter. Ceramium rubrum (Huds.) C.Ag., 1817

MGC PHYC: 1998.

a. s/n. / Conferva rubra [m. ¿Cabrera?].

b. N. 4 / Mertens [m. ¿Haenseler?].

c. s/n / Rev.: ...S. M. C. ha pasado algunas / ... [m. ¿Haenseler?].

Observaciones: Hay 22 ejemplares pegados sobre papel, donde no existe texto, más los tres anteriores.

150. Sargassum vulgare C. Ag., 1821

MGC PHYC: 1999.

a. $\square \mathrm{s} / \mathrm{n} / \ldots$ um [presuponemos Sargassum, ficha muy apolillada] vulgare / confertum

[m. Haenseler. Sin localidad].

Observaciones: Rev.: Se observan unas operaciones matemáticas.

153. Padina pavonica (L.) Lamour., 1816

MGC PHYC: 2000.

a. $\square$ s/n / Padina pavonia / (Adams.) [m. Señora inglesa. Sin localidad].

156. Ascophyllum nodosum (L.) Le Jolis, 1863

MGC PHYC: 2001.

a. $\square \mathrm{s} / \mathrm{n}$ / Fucus nodosus [m. Señora inglesa. Sin localidad].

157. Laminaria digitata (Huds.) Lamour., 1813

MGC PHYC: 2002.

a. $\square \mathrm{s} / \mathrm{n}$ / Laminaria digitata . [m. Señora. inglesa. Sin localidad].

Observaciones: Sin cauloides y sin rizoides.

158. Saccorhiza polyschides (Lightf.) Batters, 1902 MGC PHYC: 2003.

a. $\square \mathrm{s} / \mathrm{n}$ / Laminaria bulbosa ?. [m. Señora inglesa. Sin localidad].

Observaciones: Pequeño ejemplar, no obstante, completo y claramente identificable.

159. Cryptopleura ramosa (Huds.)Kylin ex L.Newton, 1931

MGC PHYC: 2004

a. $\square \mathrm{s} / \mathrm{n}$ / Nitophyllum laceratum ?.[m. Señora inglesa. Sin localidad]. 
160. Plocamium cartilagineum (L.) Dixon, 1957

MGC PHYC 2005.

a. s/n. / Plocamium cocineum [m. Señora inglesa. Sin localidad].

161. Laminaria digitata (Huds.) Lamour., 1813

MGC PHYC: 2006.

a. $\square \mathrm{s} / \mathrm{n}$ / Laminaria digitata [m. Señora inglesa. Sin localidad].

162. Plocamium cartilagineum (L.) Dixon, 1957

MGC PHYC: 2007.

a. s/n / Plocamium cocineum [m. Señora inglesa. Sin localidad].

163. Callithamnion byssoides Arnott ex Harv. in Hook., 1833

MGC PHYC: 2008.

a. s/n. / Callithamnion / byssoideum / (Arnot) [m. Señora inglesa. Sin localidad].

165. Saccorhiza polyschides (Lightf.) Batters, 1902

MGC PHYC: 2009.

a. $\square \mathrm{s} / \mathrm{n}$ / Laminaria bulbosa [m. Señora inglesa. Sin localidad].

Observaciones: Sólo existe la parte basal y algo de estipe de dos ejemplares.

G. Algas de Nápoles.

168. Jania rubens (L.) Lamouroux, 1812

MGC PHYC: 2010.

a. $\square$ s/n / Corallina rubens / Neapel / leg. et v./ V. Alfers [m.i.: ¿Alfers?].

169. Stypocaulon scoparium (L.) Kütz., 1843

MGC PHYC: 2011.

a. $\square \mathrm{s} / \mathrm{n}$ / Sphacellaria scoparia / Posilippo / Leg. et v./ V. Alfers [m.i.: ¿Alfers?]. etiqueta.

Observaciones: El ejemplar va metido en un sobrecito hecho del papel mismo de la

H. Algas de Suecia.

171. Polysiphonia violacea (Roth) Spreng., 1827

MGC PHYC: 2012.

a. $\square$ N. 1713 [m.i. tachado], 2657 / Ceramium violaceum B allochroas. / Agardh. l. c. $/ /$ e Melandia [m. Haenseler].

b. s/n/C. allochroas/violacei variet in F. vesiculoso/ad Malmo [m. ¿C. Agardh?].(Lám.

II, fig. 1 y Lám. IV, fig. 5).

173. Polysiphonia violacea (Roth) Spreng., 1827

MGC PHYC: 2013.

a. s/n. / Ceramium violaceum / Sueciae [m.i.].

b. s/n. / Ceramium violaceum / Sueciae [m.i.].

c. s/n. / Ceramium siliculosum / Sueciae [m.i.].

Observaciones: Tan sólo los ejemplares a y b corresponden a Polysiphonia.

178. Elachista fucicola (Velley) J. Areschoug, 1842

MGC PHYC: 2014.

a. N 1724 [tachado ¿Haenseler?], 2679 [m. ¿Haenseler?] Conferva fucicola / Suecia [m. ¿C. Agardh?].

I. Algas de Helgoland.

182-3. Callithamnion corymbosum (Sm.) Lyngb., 1819 MGC PHYC: 2015. 
a. s/n. / Callithamnion corymbosum / Helgoland, Calverdons / August 65 / leg. et v. P. Magnus [m. P. Magnus].

b. s/n. / Callithamnion / corymbosum / Dubnuvdand [ilegible] Helgoland / 20/IX. 65 [m. ¿P. Magnus?].

184. Rhodomela confervoides (Huds.) Silva, 1952

MGC PHYC: 2016.

a. s/n. / Rhodomela subfusca/Garbphyphund [ilegible]/ August. 1865 [m. ¿P. Magnus?].

185. Fucus serratus L., 1753

MGC PHYC: 2017.

a. $\square$ s/n. / Fucus serratus / Helgoland [ésto es legible, pero después aparecen dos palabras ilegibles correspondientes posiblemente a una determinaciòn màs especìfica de la localidad] / August. 65 l. et v. P. Magnus [m. Magnus].(Lám. IV, fig. 4).

186. Laminaria saccharina (L.) Lamouroux, 1813

MGC PHYC: 2018.

a. s/n / Fucoideae Lamouroux / Laminaria saccharina Lamx. / legit: Correns auf [ilegible] / Helgoland August 51 / O. Jaenicke / [m. ¿Correns?, ¿Jaenicke?] (Lám. II, fig. 4). Observaciones: El ejemplar relativamente joven, está sujeto al pliego mediante tirillas de papel adhesivo.(Lám. V, fig. 3).

187. Halidrys siliquosa (L.) Lyngb., 1819

MGC PHYC: 2019.

a. s/n. / Halidrys siliquosa / Helgoland / August. 1865 / 1. et v.: P. Magnus [m. Magnus].(Lám. II, fig. 3 y Lám. V, fig. 1).

188. Enteromorpha intestinalis (L.) Link in Nees, 1820

MGC PHYC: 2020.

a. s/n. / Enteromorpha intestinalis // Helgoland [m. ¿Magnus?].

189. Membranoptera alata (Huds.) Stackh., 1809

MGC PHYC: 2021.

a. s/n. / Delesseria alata / Helgoland / August. 1865 l. et v. P.Magnus [m. Magnus].

(Lám. II, fig. 2 y Lám. V, fig. 2).

190 y 191 Dictyota dichotoma (Huds.) Lamour., 1809

MGC PHYC: 2022.

a. s/n. / Dictyota dichotoma [m. ¿Magnus?].

b. s/n. / Dictyota dichotoma ... [ilegible] / Helgoland / 28/VIII/65 [m. ¿Magnus?].

192. Halarachnion ligulatum (Woodw.) Kütz., 1843

MGC PHYC: 2023.

[m. Magnus].

a. s/n. / Halimenia ligulata / Helgoland ... [ilegible] August, 1865 l. et v. P. Magnus

193. Polysiphonia nigrescens (Huds.) Grev. in Hoot., 1833.

MGC PHYC: 2024.

a. s/n / Polysiphonia nigrescens [escrito a lápiz] / Helgoland [m. ¿Magnus?].

194. Ceramium rubrum (Huds.) C. Agardh, 1817

MGC PHYC: 2025.

a. s/n. / Ceramium rubrum / Helgoland ... [continúa letra ilegible, ¿referente a la localidad?] / August, 1865 // l. et v. P. Magnus [m. Magnus].

195. Furcellaria lumbricalis (Huds.) Lamour., 1813.

MGC PHYC: 2026. 
Magnus].

a. $\square$ s/n. / Furcellaria fastigiata / Helgoland / August, 1865 // l. et v.: P. Magnus [m.

197. Ahnfeltia plicata (Huds.) Fr., 1836.

MGC PHYC: 2027.

a. $\square$ s/n. / Sphaerococcus plicatus / Helgoland / August, 1865 / l.et v.: P. Magnus [m. P. Magnus].Rev.: Figura un escrito donde se puede apreciar una fecha, probablemente el 8.II.1865; lo que implica que se utilizara para hacer la etiqueta, un trozo de carta, nota, o algo similar.

198. Chondrus crispus Stackh., 1797.

MGC PHYC: 2028.

a. $\square$ s/n. / Chondrus crispus / Helgoland / August, 1865 / 1. et v.: P. Magnus [m. P. Magnus]. Rev.: Al igual que en el caso anterior figura escrito, probablemente parte también del mismo trozo de carta o nota que comentábamos anteriormente.

199. Laminaria saccharina (L.) Lamour., 1813.

MGC PHYC: 2029.

a. $\square$ s/n. / Laminaria phyllitis / Helgoland / August, 1865 / l. et v.: P. Magnus [m. P.

Magnus]. Rev.: Probablemente, la etiqueta es un trozo de la misma carta que las anteriores.

200. Chondria dasyphylla (Woodw.) C. Agardh, 1822 MGC PHYC: 2030.

a. s/n. / Laurencia dasyphylla / tetrasporen- ... [palabra ilegible] / Helgoland, ... [¿Vurblygius?] / August, 1865 / 1. et v.: P. Magnus [m. P. Magnus].

201. Scytosiphon lomentaria (Lyngb) Link., 1833.

MGC PHYC: 2031

a. s/n. / Scytosiphon lomenta- / rius ... [ilegible] /28/IX/65 / Helgoland [m. ¿Magnus?].

202. Polysiphonia urceolata (Lightf. ex Dillwyn) Grev., 1824.

MGC PHYC: 2032.

a. s/n./ Polysiphonia urceolata / Helgoland / l. et v.: P. Magnus [m. P. Magnus].

205. Plocamium cartilagineum (L.) Dixon, 1957.

MGC PHYC: 2033. Magnus].

a. s/n./ Plocamium coccineum / Helgoland / August, 1865 / 1. et v.: P. Magnus [m. P.

Observaciones: A través de una figura $(\mathrm{O})$ da nota del estado reproductor (tetrásporas).[Está también escrita a lápiz].

206. Brongniartella byssoides (Good. et Woodw.) F. Schmitz, 1893.

MGC PHYC: 2034.

a. s/n. / Polysiphonia bissoides / Helgoland ... [varias palabras ilegibles] / August, 1865 / 1. et v.: P. Magnus [m. P. Magnus].

207. Polysiphonia elongata (Huds.) Spreng., 1827.

MGC PHYC: 2035.

a. s/n. / Polysiphonia elongata / ... [ilegible] / Helgoland / 9/X/65 [m. ¿Magnus?]

208. Cladostephus spongiosus (Huds.) C. Agardh, 1828.

MGC PHYC: 2036.

P. Magnus].

a. s/n./ Cladostephus spongiosus / Helgoland / August, 1865 / l. et v.: P. Magnus [m.

J. Algas extranjeras

209. Odonthalia dentata (L.) Lyngb., 1819.

MGC PHYC: 2037.

a. $\square$ N. 2555 / Fucus dentatus L. [Fucus aparece débilmente tachado, por Señora 
inglesa] / Odonthalia / a D. Schou... [falta un trozo] / e mari n... [ficha rota, carcomida] [m. Haenseler].

\section{Phyllophora crispa (Hudson) Dixon, 1964}

MGC PHYC: 2038.

a. $\square$ N. 1162 [m.i. tachado], 1671 [m.i. tachado], 2612 / Fucus rubens L. / F. fronde membranacea dichotoma catenato - / prolifera ramis elllipticis ; ramulis apice/dilatatis bifidis. Herb. Linn. Turn. 1. c. p. $216 / /$ a D. Schousb. e mari septentrionale // in mari gaditano a D. Cabrera [tachado] [m. Haenseler].

211. Enteromorpha linza (L.) J. Ag., 1883 MGC PHYC: 2039.

a. $\square \mathrm{s} / \mathrm{n}$. / Ulva lactuca [no es Ulva] ... [ilegible] / ... [dos palabras ilegibles ¿Alfers?] [m. ¿Magnus?. La letra sin embargo se parece a las anteriores de Helgoland].

213. Enteromorpha intestinalis (L.) Link in Nees, 1820 MGC PHYC: 2040.

a. $\square \mathrm{s} / \mathrm{n}$. / Enteromorpha intestinalis / ... [ilegible] [¿Hazsburgo?] / ... [ilegible] [¿Neukirhen?] / Sept. 69 / ... [¿Dr. Sauden?].[m.i.].

214. Gymnogongrus griffithsiae (Turner) G. Martius, 1828 MGC PHYC: 2041.

Haenseler].

a. $\square$ N. ... [etiqueta rota] / Fucus fastigiat... [roto] / ex América a D. Cabrera [m.

215. Delesseria sanguinea (Huds.) Lamour., 1813 MGC PHYC: 2042. Agardh?].

a. $\square$ N. 2617 [m. Haenseler] / Fucus / sanguineus / e mari septentrionale [m. ¿C.

216. Furcellaria lumbricalis (Huds.) Lamour., 1813.

MGC PHYC: 2043.

66/ Thzhiehl [m.i.].

a. s/n. / Furcellaria fastigiata / ... [ilegible] / Brand von Linnowitz auf Ugedom Juli

217. Porphyra umbilicalis (L.) J. Ag., 1883

MGC PHYC: 2044.

a. s/n. / Porphyra vulgaris [m.i.].

218. Delesseria sanguinea (Huds.) Lamour., 1813

MGC PHYC: 2045. ilegibles].[m.i.].

a. s/n./ Delesseria sanguinea Lamour / ... [varias palabras ilegibles] / ... [palabras

219. Fucus vesiculosus L., 1753

MGC PHYC: 2046.

a. $\square \mathrm{s} / \mathrm{n}$. / Fucus vesiculosus / ... [dos palabras ilegibles] / 26.8.60 ... [¿Lauder?] [m.i.].

K. Algas de Noruega.

221. Corallina officinalis L., 1758

MGC PHYC: 2047.

a. s/n. / Corallina officinalis / Robuste Form / Norvergen [¿Kloppen bei Strugesund?] /27/7/1872 [m. P. Magnus].

Observaciones: Son buenos ejemplares, los cuales van metidos en una especie de sobre, hecho a mano, y donde en el frente figura el texto: identificación, localidad y fecha.

222. Desmarestia aculeata (L?) Lamour., 1813

MGC PHYC: 2048.

a. $\square$ N. 2527 / Fucus aculeatus / a D. Schousboe e Norvega [m. Haenseler]. 
223. Ptilota plumosa (Huds.) C. Ag., 1817

MGC PHYC: 2049.

a. $\square$ N. 2607 / Fucus [tachado, con trazo fino] plumosus L. / Ptilota plumosa [m. Señora inglesa] // a D. Schousb. / e mari norvegico [m. Haenseler].

L. Algas de Bandoran, Cork Harbour e Ireland

226. Hypnea musciformis (Wulfen) Lamour., 1813

GR: 608 b.

Señora inglesa].

a. s/n. Hypnea purpu [tachado] purpurascens / (Huds.) / Bandoran / July 1850 [m.

231. Cryptopleura ramosa (Huds.) Kylin ex L. Newton, 1931

GR: 521.

a. s/n. / Nitoz [tachado] / Nitophyllum laceratum / Gmel. / Cork [m. Señora inglesa].

232. Plumaria elegans (Bonnem.) F.Schmitz, 1889

GR: 433 b.

a. s/n. Ptilota / P.[ tachado] / sericea / Gmel. / Cork [m. Señora inglesa].

235. Callophyllis laciniata (Huds.) Kütz., 1843

GR: 547 b.

a. s/n. / Rhodymenia / laciniata / Ireland [m. Señora inglesa].

238. Furcellaria lumbricalis (Huds.) Lamour., 1813

GR: 572.

a. s/n./ Furcellaria / fastigiata / (Hudson) / 89 [con lápiz] / Cork Harb. / May 1850 [m. Señora inglesa].

242. Laurencia pinnatifida (Huds.) Lamour., 1813

GR: 467.

Señora inglesa].

a. s/n. / Laurencia / pinnatifida / Gmel. 43 [con lápiz] / Cork Harb. / May 1850 [m.

Observaciones: Ver el 59 de Málaga. Hay cuatro folios, uno de ellos se corresponde a este ejemplar.

\section{RESULTADOS Y CONCLUSIONES}

De los 242 pliegos hemos podido revisar 148 ejemplares, de ellos 27 están en Granada, junto con 26 de los 29 de Clemente. Luego de los 96 táxones recopilados, 60 eran de Rodofíceas, 27 Feofíceas y 9 Clorofíceas. Es pues, que en el MGC.PHYC, tenemos 121 pliegos identificados en 85 táxones (53 Rodofíceas, 24 Feofíceas y 8 Clorofíceas.

Creemos que el material que poseemos de Halymenia floresia (Clemente) $\mathrm{C}$. Agardh, 1822 de Cádiz («in mari gaditano a D. Cabrera») nº 33 de esta colección del HSMC, sub. Fucus floresius Clemente y cuya etiqueta original aparece con la descripción del ejemplar según el «ensayo de la vid» p. 312 (ver lámina VI) habría que considerarlo un neótipo, ya que Codomier (1974: 3) no nos da referencias al respecto.

Hay que decir también que motivado por el pliego no 34 de Cádiz, Fucus flavus L., hemos descubierto que el $F$. tournefortii de Lamouroux, 1805, es prioritario al $F$. flavus Clemente, 1804 (ya que este último año es erróneo) por lo que revalidamos el nombre Zonaria tournefortii (Lamouroux) Montagne, 1846, en contra de lo que propone Nizamuddin (1981: 32). 


\section{CATÁLOGO FLORÍSTICO}

A continuación damos el catálogo florístico ordenado alfabéticamente, con las localidades y el número de los pliegos correspondientes al HSMC.

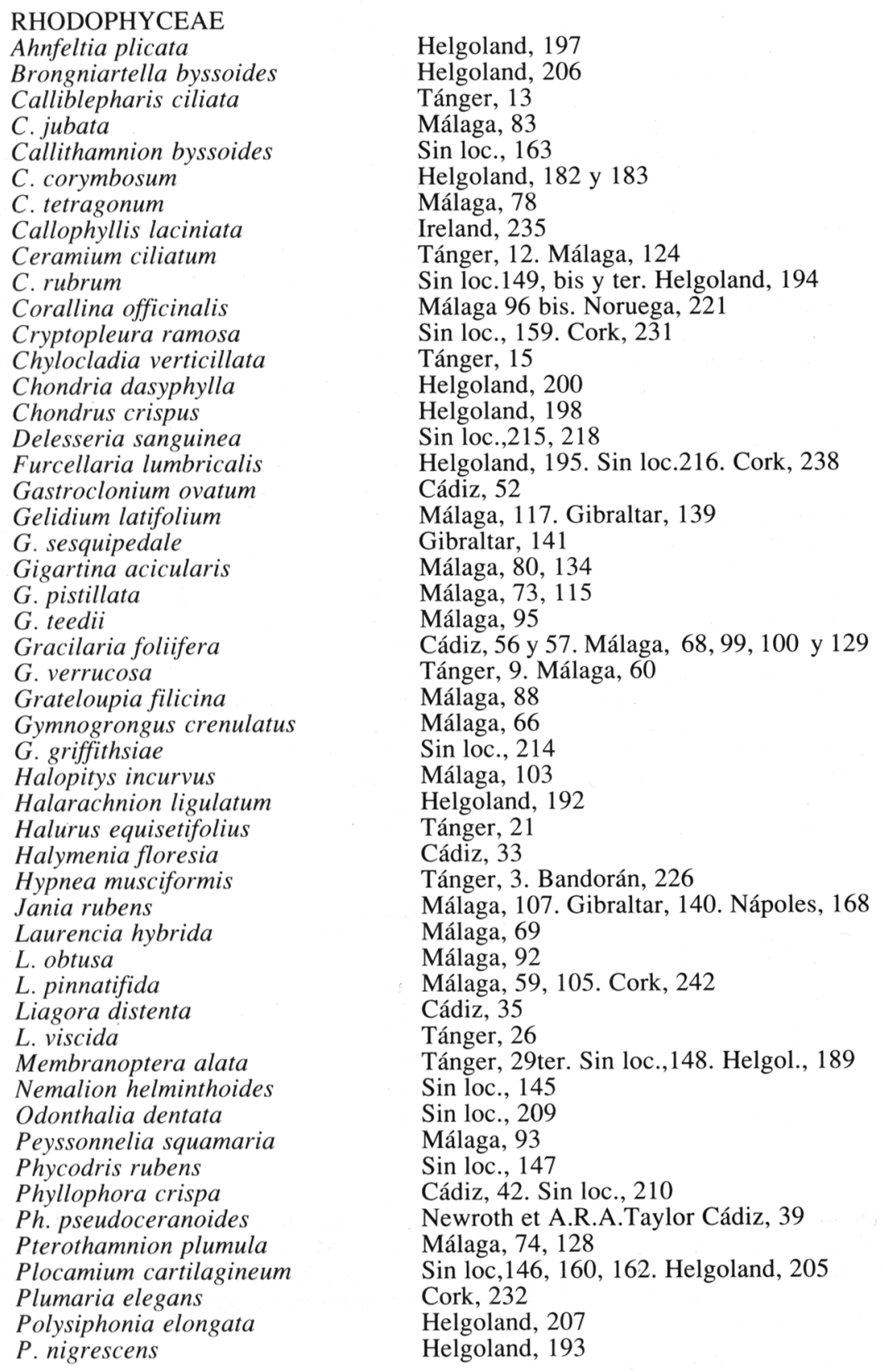


P. urceolata

$P$. violacea

Porphyra umbilicalis

Pterocladia capillacea

Pterosiphonia complanata

Ptilota plumosa

Rhodomela confervoides

Rytiphloea tinctoria

Schyzimenia dubyi

\section{PHAEOPHYCEAE}

Ascophyllum nodosum

Cladostephus spongiosus

fo. verticillatus

Colpomenia sinuosa

Cystoseira nodicaulis

C. tamariscifolia

Desmarestia aculeata

Dictyopteris polypodioides

Dictyota dichotoma

Ectocarpus fasciculatus

Elachista fucicola

Fucus serratus

$F$. vesiculosus

Halidrys siliquosa

Halopteris filicina

Laminaria digitata

L. saccharina

Myriotrichia filiformis

Padina pavonica

Petalonia fascia

Pylaiea littoralis

Saccorhiza polyschides

Sargassum vulgare

Scytosiphon lomentaria

Stypocaulon scoparium

Taonia atomaria

Zonaria tournefortii

\section{CHLOROPHYCEAE}

Bryopsis plumosa

Caulerpa racemosa

Codium vermilara

Enteromorpha compressa

E. intestinalis

E. linza

E. ramulosa

Ulva lactuca L. fo. cribosa

U. rigida
Helgoland, 202

Suecia, 171, 173

Sin loc., 217

Málaga, 111

Málaga, 65, 77, 98

Noruega, 223

Helgoland, 184

Cádiz, 46

Málaga, 72, 116

Sin loc., 156

Helgoland, 208

Málaga, 132

Málaga, 91 bis

Málaga, 61

Málaga, 63, 96

Noruega, 222

Tánger, 19. Málaga, 82

Cádiz, 51. Málaga, 106. Helgol., 190, 191

Cádiz, 40

Suecia, 178

Cádiz, 142. Helgoland, 185

Cádiz, 45. Málaga, 135. Sin loc., 219

Tánger, 28. Helgoland, 187

Málaga, 62

Sin loc., 157, 161

Helgoland, 186, 199

Sin loc., 143

Málaga, 102. Sin loc. 153

Málaga, 64,86

Sin loc., 144

Sin loc., 158, 165

Sin loc., 150

Helgoland, 201

Gibraltar, 138. Nápoles, 169

Cádiz, 36

Cádiz, 34

Málaga, 76

Tánger, 24

Málaga, 101

Cádiz, 47

Helgoland, 188. Sin loc., 213

Sin loc., 211

Cádiz, 50

Tánger, 8

Málaga, 91

Dedicado. A D. Modesto Laza Palacios, ilustre botánico malagueño y presidente de honor del III Simposio Nacional de Botánica Criptogámica (Málaga, 9-12 octubre de 1978) y a D. Luis Bellón Uriarte representante nacional en el First International Seaweed Symposium (Edimburgo, julio 1952), que a través de su obra y su biblioteca me inició en la ciencia del macrofitobentos. 


\section{BIBLIOGRAFÍA}

BARRA, A. y G. LÓPEZ GONZÁLEZ -1984- Tipificación de los táxones del género Narcissus (Amaryllidaceae) descritos por Linneo. Anales Jard. Bot. Madrid, 40(2): 345-360.

BELLÓN, L. - 1940- Nota sobre un herbario de algas de Málaga, de D. Simón de Rojas Clemente y Rubio. Boll. Inst. Esp. Oc.. Notas y resúmenes, 102: 1-29.

BELLÓN, L. -1942-Las algas de la «Flora Boetica» inédita de Clemente. Boll. Inst. Esp. Oc., Notas y resúmenes, 110: 1-93.

BUENO, A.G., M. GUTIÉRREZ BUSTILLO y C. NAVARRO -1985- La contribución a la Botánica de B. Lázaro Ibiza (1858-1921): Indice de novedades nomenclaturales (plantas vasculares) y tipificación de táxones específicos. Lazaroa, 8: 353-370.

BURDET, H.M., A. CHARPIN \& F. JACQUEMOUD -1981- Types nomenclaturaux ibériques décrits par Boissier ou Reuter. I. Gymnospermes à Graminées. Candollea, 36(2): 543-584

CANDOLLE, DE, A.P. -1818-Regni vegetabilis systema naturae sive ordines, genera et species, plantarum secundum methodi naturalis normas digistarum et descriptarum. 1: 1-564. París.

CARRILLO, J.L., M.D. RAMOS y J. CASTELLANOS -1984-Catálogo de manuscritos. Imprenta Universidad de Málaga. $161 \mathrm{pp}$.

CLEMENTE Y RUBIO, S.R. -1807-Ensayos sobre las variedades de la vid común que vegetan en Andalucía, con un índice etimológico y tres listas de plantas en que se caracterizan varias especies nuevas. Lista de algas p. 308-322. Imprenta Villalpando, Madrid.

CODOMIER, L. -1974- Recherches sur la structure et le développement des Halymenia C. Agardh (Rhodophycée, Cryptonémiales) des côtes de France et de la Méditerranée. Vie et milieu, 24 (1A) $1-42$.

COLMEIRO, M. -1858-La botánica y los botánicos de la Península Hispano-lusitana. Estudios bibliográficos y biográficos. Imp. Rivadeneira. $216 \mathrm{pp}$. Madrid.

CONDE, F. -1981-Estudio sobre las algas bentónicas del litoral de la provincia de Málaga. Tesis doctoral. Universidad de Málaga. 374 pp.

CONDE,F. -1984-Catálogo de las algas macrobentónicas marinas de Málaga.Acta Bot.Malacitana, 9: $47-78$.

FLORES MOYA, A., J.J. VERA GONZÁLEZ y F. CONDE -1989- Contribución a la corología de las macroalgas marinas bentónicas del litoral malagueño: I. Acta Bot. Malacitana, 14: 199201 .

GONZÁlLEZ FRAGOSO, R. -1886- Plantas marinas de la costa de Cádiz. An. Soc. Esp. Hist. Nat., 15: 117-130.

LAZA, M. -1942- Nota sobre un herbario de plantas andaluzas de D. Simón de Rojas Clemente y Rubio. Bol. Real Soc. Esp. Hist. Nat., 40: 263-298.

LAZA, M. - 1945-Estudios sobre la flora y la vegetación de las Sierras Tejeda y Almijara. An. Jard. Bot. Madrid, 6(2): 217-370.

LINNAEUS, C. -1781- Supplementum plantarum ... (16) + 467 pp. Holmiae. (No visto).

NAVARRO, F. -1955- Don Luis Bellón Uriarte (1897-1954) Bol. Real Soc. Esp. Hist. Nat., Sec. Biológica, 53: 13-20.

NIZAMUDDIN, M. -1981- Contribution to the marine algae of Libya. Dictyotales. Bibliotheca Phycologica, 54: 1-122. Cramer.

PÉREZ-CIRERA,J.L., J.CREMADEe I. BARBARA-1989-Grateloupia lanceola (Cryptonemiales, Rhodophyta) en las costas de la Península Ibérica: Estudio morfológico y anatómico. Lazaroa, 11: 123-134.

SEOANE, J. -1965- Estudios sobre las algas bentónicas en la costa Sur de la Península Ibérica (litoral de Cádiz). Inv. Pesq., 29: 3-216.

SEOANE, J. -1969-Algas bentónicas de Menorca en los herbarios Thuret-Bornet y Sauvageau del Museum National d'Histoire Naturelle de Paris. Inv. Pesq., 33(1): 213-260.

TÉLLEZ, R. y M. ALONSO -1952-Los trigos de la Ceres hispanica de Lagasca y Clemente. INIA, 516 pp. Madrid.

(Aceptado para su publicación en Julio de 1991)

Dirección del autor: Departamento de Biología Vegetal. Facultad de Ciencias. Universidad de Málaga. 29080 Málaga. 


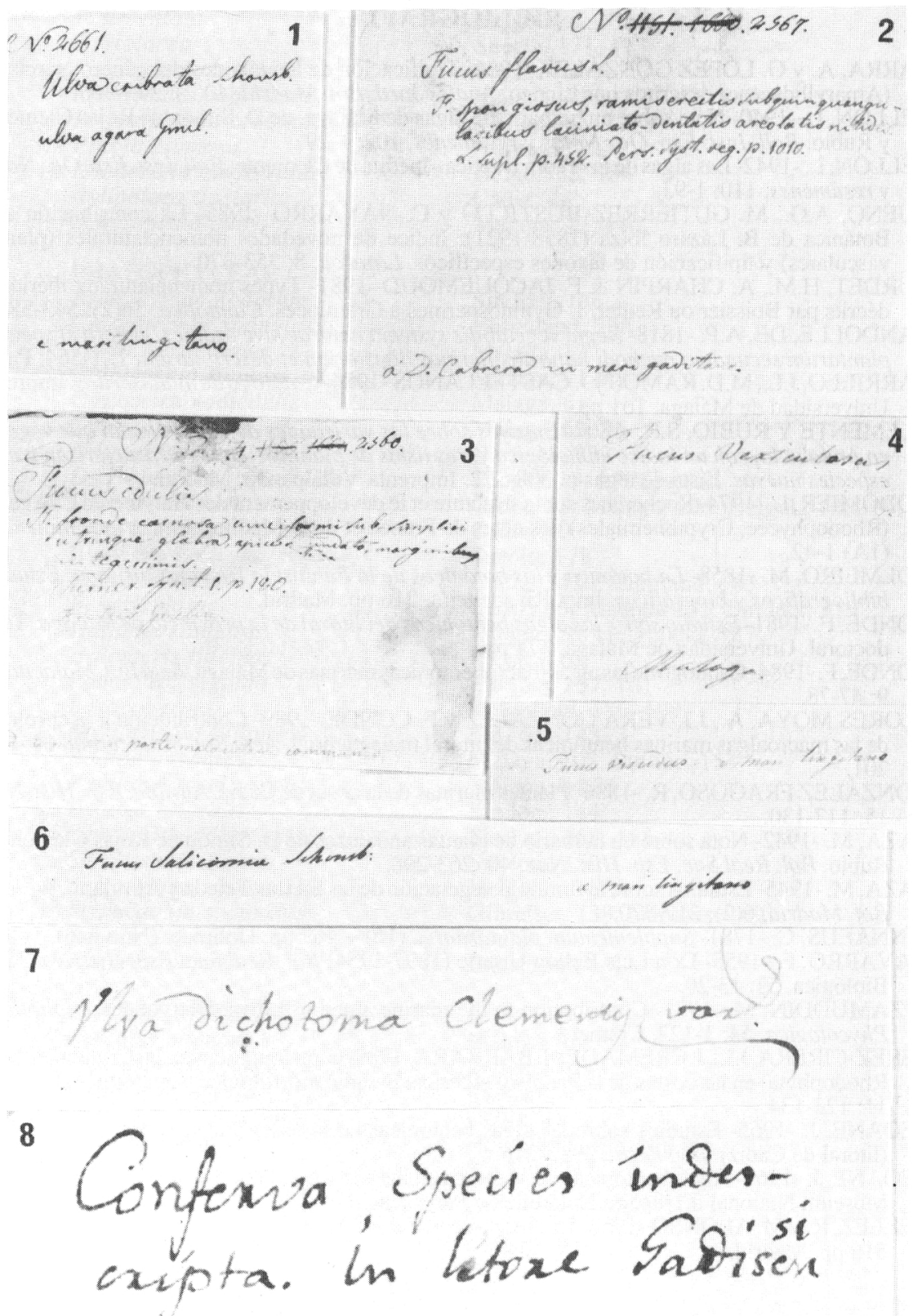

Lámina I. Diferentes tipos de fichas con letras de distintos autores. Figs. 1 a 3, con letra de Haenseler. Fig. 4, con letra de Prolongo. Fig. 5 y 6 , con letra de Schousboe. Fig. 7 y 8 , con letra de Cabrera. 
Colección de algas de la Sociedad Malagueña de Ciencias

51

1

C. Alloclua an.

2

seicrace wanef

in J. Veficuloto. ail thatoms-

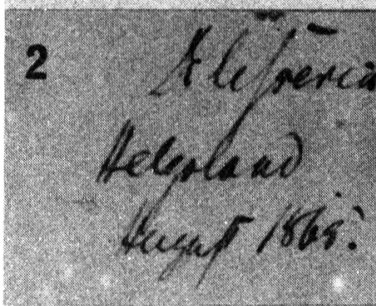

3

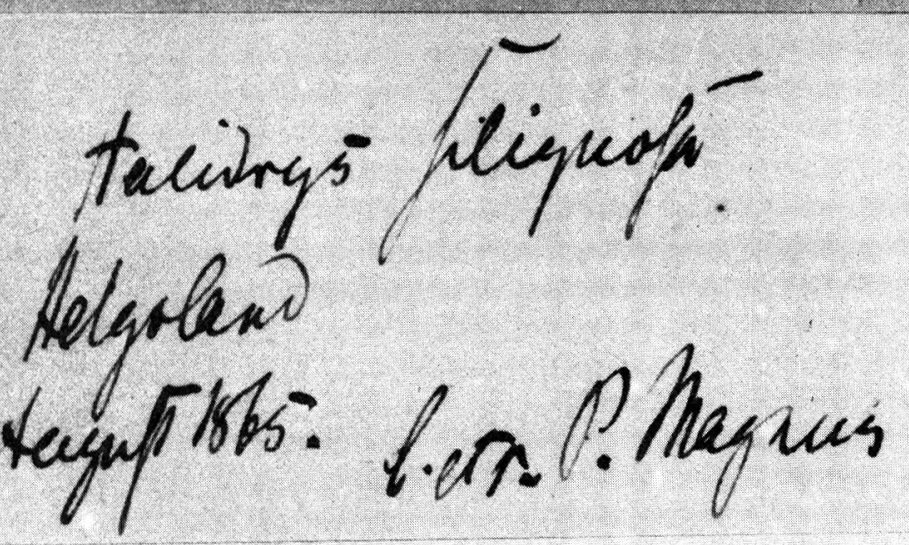

4

Pucilear sámencomer.

Saminaria sacilaxisua stam.

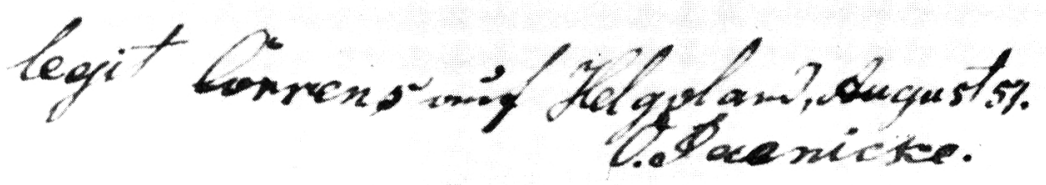

Lámina II. Fichas y letras de autores. Fig. 1, con letra probable de C. Agardh. Fig. 2 y 3, con letra de P. Magnus. Fig. 4, con letra de Córrens o/y Jaenicke. 

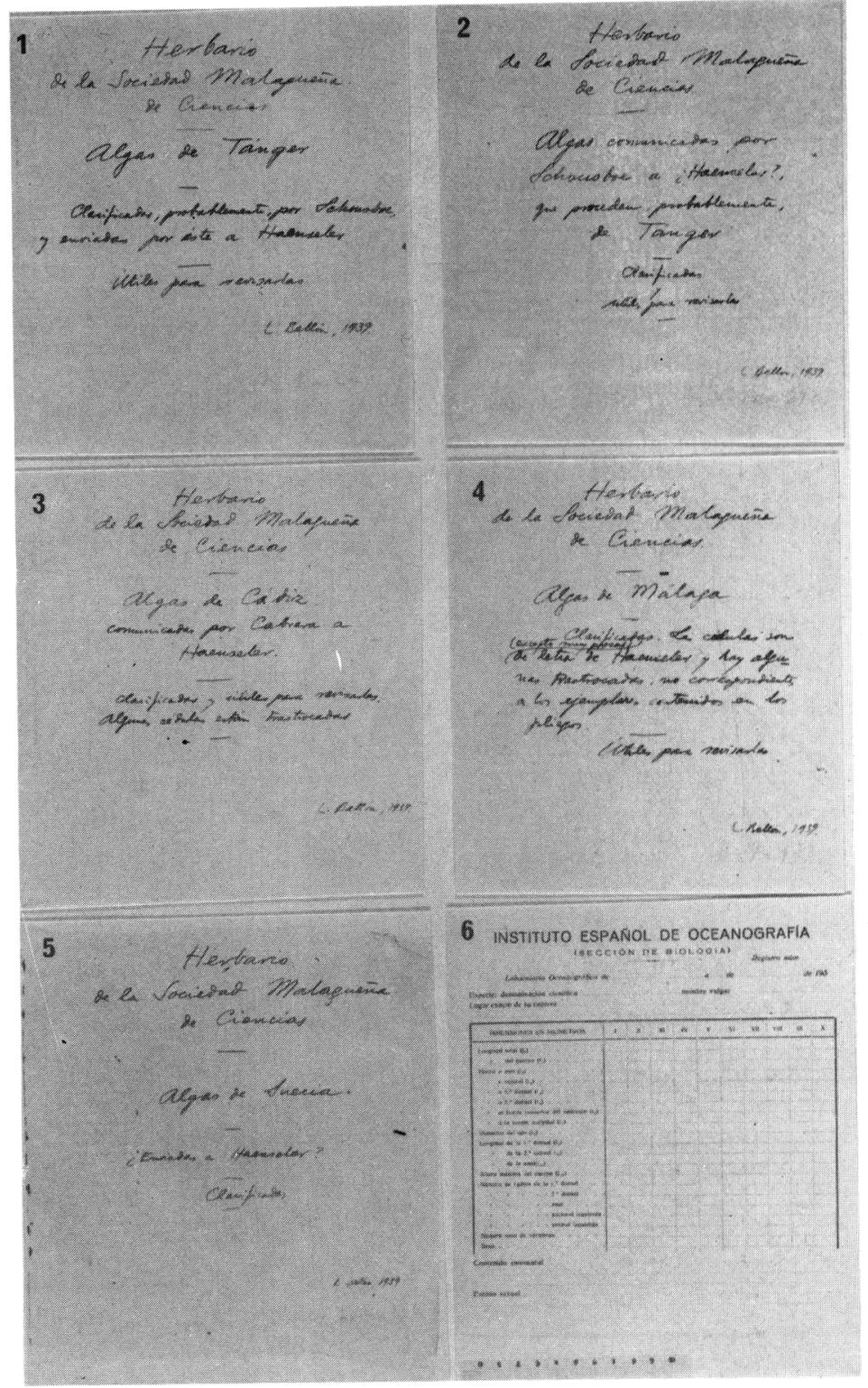

Lámina III. Reverso (figs. 1 a 5) y anverso (fig. 6) de algunos de los papeles separadores que con letra de L. Bellón, figuraban los distintos pliegos de las diversas procedencias y que servían para diferenciarlos, por grupos de localidades unos de otros y en que se pueden leer quienes eran los autores que las enviaban. En las figuras 1 a 5 , se puede observar debajo de L. Bellón 1939, la cantidad de pliegos que separaría cada uno de aquellos papeles. Fig. 1, n⿳ 1 a 26; fig. 2, no 27 a 29 ter; fig. 3, n 30 a 57; fig. 4, n 89 a 137; fig. 5, 170 a 181 . Están escritos en lápiz rojo, menos visible que lo otro que figura en tinta negra. La escala de la fig. 6 va en $\mathrm{cm}$, y es igualmente válida para las otras 5. 


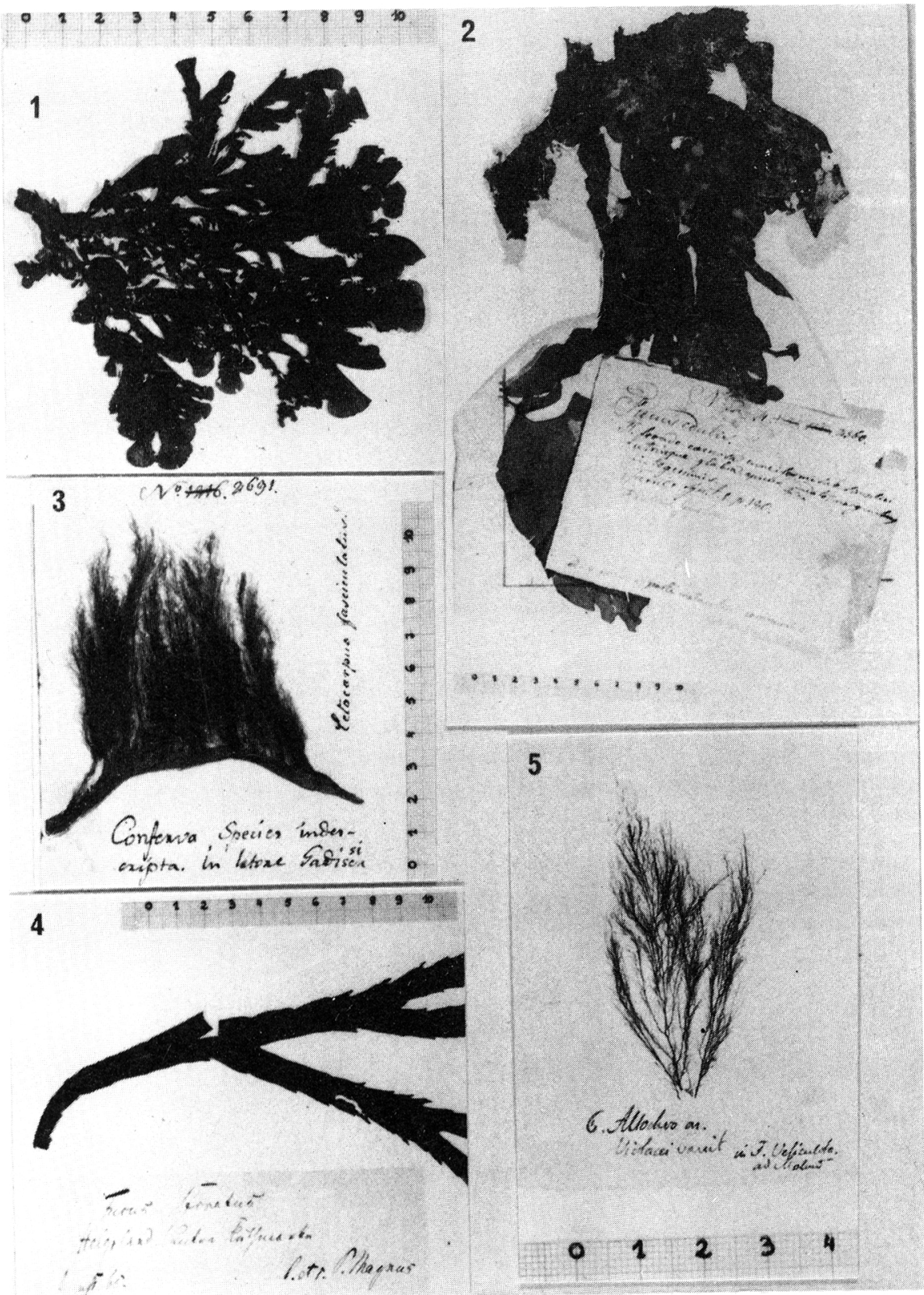

Limma IV. Ejemplos de diferentes pliegos, en donde muchos de ellos se puede observar fijo el ejemplar sobre el papel donde figura la identificación. En algunos otros, los ejemplares van sueltos y las cédulas de identificación van en etiquetas que pueden tener distintos tamaños: Fig. 1, Zonaria tournefortii (Lam.) Montagne, pliego no 34; Fig.2, Schizymenia dubyi (Chauvin ex Duby) J.Ag., pliego n"1 16; Fig.3, Ectocarpus fasciculatus? Harvey, pliego n* 40; Fig. 4, Fucus serratus L., pliego n" 185; Fig. 5, Polysiphonia violacea (Roth) Spreng., pliego $n^{\circ} 171$. 

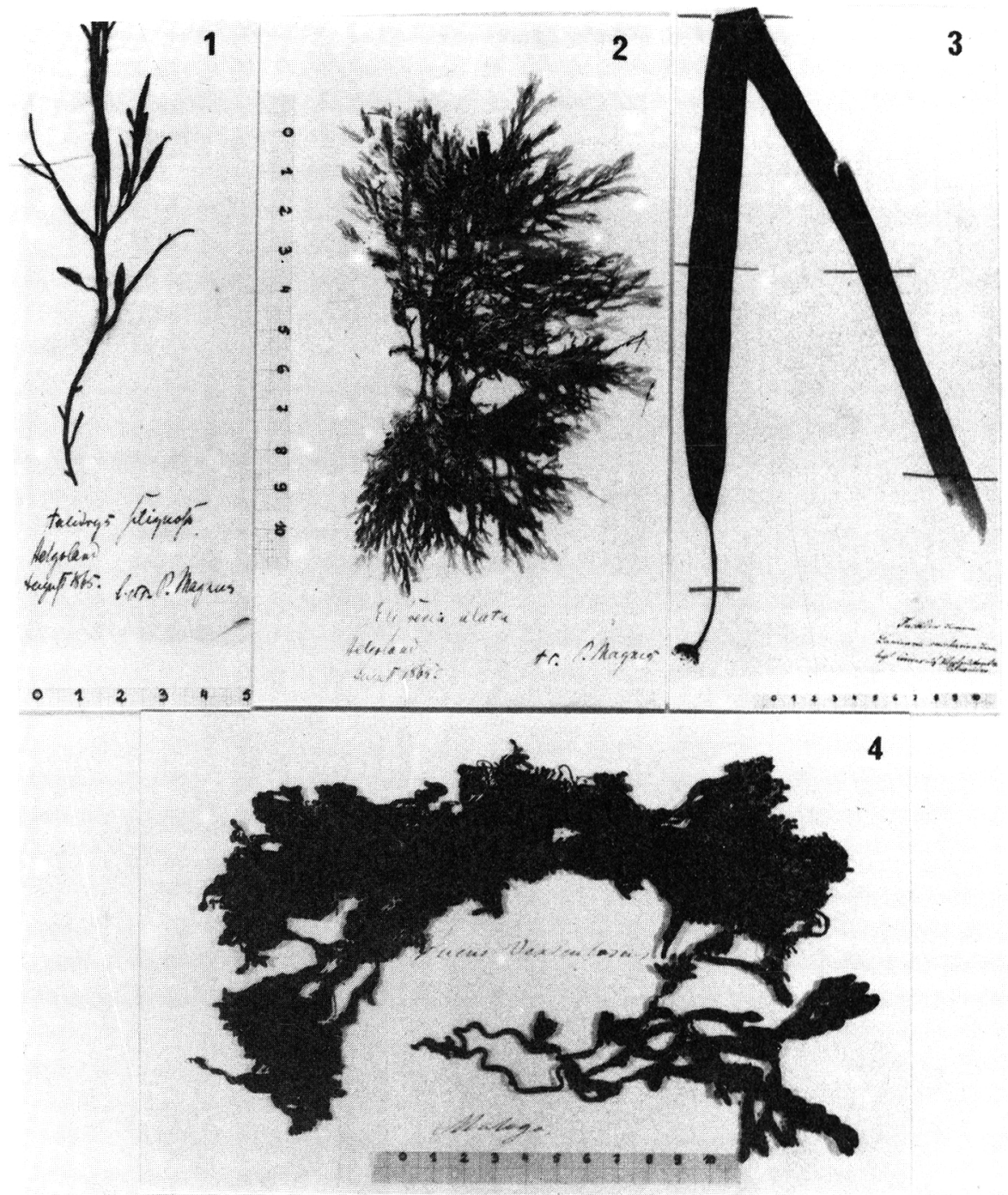

Lámina V. Idem lámina IV. Fig. 1, Halidrys siliquosa (L.) Lyngb., pliego n" 187; Fig. 2, Membranoptera alata (Huds.) Stackh., pliego no 189; Fig. 3, Laminaria saccharina (L.) Lam., pliego nº 186; Fig. 4, Fucus vesiculosus L., pliego nº 135. 

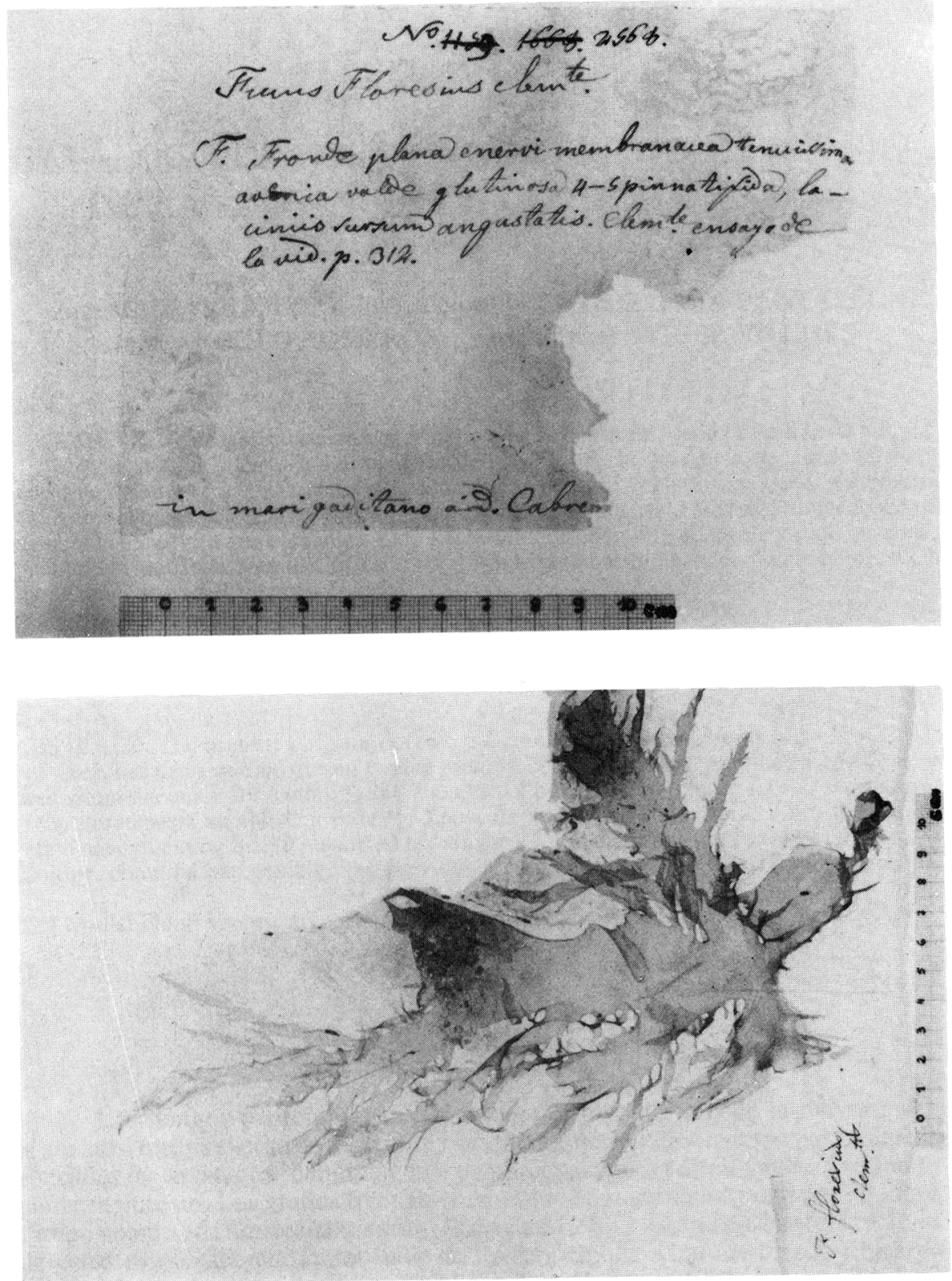

Lámina VI. Halymenia floresia (Clemente) C. Ag., sub. Fucus floresius, de Cádiz, no 33. Se puede observar en la parte superior la etiqueta original y con letra de Haenseler la descripción del ejemplar. En la parte inferior y donde figura el especimen, en la parte derecha, aparece el basiónimo con letra desconocida. Las escalas de las distintas figuras van en $\mathrm{cm}$. 